\title{
ATLAS L1 Muon Trigger Upgrade with sTGC: Design and Performance
}

\author{
Davide Gerbaudo*† \\ University of California, Irvine \\ E-mail: gerbaudo@cern.ch
}

\begin{abstract}
We describe the upgrade of the ATLAS forward Level 1 (L1) muon trigger planned for the LHC run with luminosity above $2 \times 10^{34} \mathrm{~cm}^{-2} \mathrm{~s}^{-1}$. This upgrade, which aims at suppressing the fake muon triggers from non-pointing tracks, foresees the installation of a New Small Wheel (NSW) detector in the endcap region. This region of the detector will be instrumented with small-strip Thin Gap Chambers (sTGC) that will allow to keep the L1 muon trigger rate below $25 \mathrm{kHz}$. This rate suppression is realized with a two-step trigger system: first, an ultra-fast pad trigger defines the regions of interest containing potential high- $p_{T}$ muon candidates; second, an accurate track measurement is performed with precision readouts from the sTGC strips, providing the required $1 \mathrm{mrad}$ angular resolution.

The new, sTGC-based, L1 muon trigger is reviewed. A description of the sTGC detector as well as of its readout system is given. The first results from the simulation of this new trigger system are presented. These studies show that the pad-trigger system is almost $100 \%$ efficient, and that the angular resolution achieved with the sTGC measurements strips is better than $1 \mathrm{mrad}$, giving an overall sTGC trigger efficiency close to $93 \%$. The system performance with the pileup and background rates expected for the high-luminosity LHC are presented as well, including an estimate of the fake muon track rate.
\end{abstract}

The European Physical Society Conference on High Energy Physics -EPS-HEP2013

18-24 July 2013

Stockholm, Sweden

\footnotetext{
* Speaker.

${ }^{\dagger}$ On behalf of the ATLAS Collaboration.
} 


\section{Motivation}

The ATLAS trigger system is organized in three levels, with increasing degree of detail and accuracy. The current level 1 (L1) forward muon trigger is based on the track $p_{T}$ measured in the big-wheel detectors. At L1, the endcap region has a large false positive (fake) rate due to the production of secondary particles in the endcap material, as illustrated in figure 1. The current fake rate becomes unsustainable when extrapolated to the LHC running conditions for 2018. The introduction of the New Small Wheel detector (NSW), and its inclusion in the L1 trigger, aims at reducing this fake rate without increasing the minimum transverse momentum required to trigger muons at L1.

Without reducing the fake rate, the L1 $p_{T}$ threshold would need to be raised, reducing the reconstruction efficiency of many critical physics analyses: Higgs boson, B-physics, SUSY and exotic searches. physics analyses searches. For example, an increase from $p_{T}>20 \mathrm{GeV}$ to $p_{T}>$ $40 \mathrm{GeV}$ would reduce the number of $W H \rightarrow \mu v b \bar{b}$ recorded events by $34 \%$, and the number of $W H \rightarrow \mu \nu W^{+} W^{-}$events by $20 \%$. The reduction would be even more dramatic if the forward region were to be excluded from the $\mathrm{L} 1$ muon trigger decision.

\section{Requirements and Challenges}

Fake triggers correspond to the tracks of charged particles that do not originate from the interaction point, but instead from the toroid region. The NSW will require at L1 a track match between the small wheel and the big wheel. In order to perform this match, the NSW track segment must be reconstructed within $1 \mu \mathrm{s}$ after the collision with $1 \mathrm{mrad}$ angular resolution. In this region, close to the beampipe, the detector must tolerate hit rates up to $15 \mathrm{kHz} \mathrm{cm}^{-2}$, as well as the consequent radiation damage.

The NSW will use small-strip Thin Gas Chamber (sTGC) detectors. The sTGC detector is a gas ionization chamber ( $2.8 \mathrm{~mm}$-thick gas volume filled with $\mathrm{CO} 2+\mathrm{n}$-pentane) operated at high voltage $(2.85 \mathrm{kV})$ with multiple readouts. Large $(\sim 10 \mathrm{~cm} \times 80 \mathrm{~cm})$ pads provide a very fast signal that can be used to identify the bunch crossing as well as a region of interest within each sector. The signal from readout strips $(3.2 \mathrm{~mm}$ pitch) can then be processed to provide a more accurate position measurement. Wires $(1.8 \mathrm{~mm}$ pitch) can be used to improve the offline precision. The precision required at $\mathrm{L} 1$ is achieved by computing the charge centroid from the strip readouts; however, conventional ADCs are too slow so alternative schemes were developed (peak-sensitive measurement and time-over-threshold conversion).

\section{Design and Implementation}

Eight sTGC layers, grouped in two 4-layer multiplets with one micromegas (MM) detector in between, will provide a redundant, fast, and accurate measurement of the track. The MM detector will improve the offline muon $p_{T}$ measurement.

A pad trigger is generated when three out of four (3/4) or four out of four (4/4) coincident pads have signals on a 4-layer multiplet. Pads are staggered to reduce the solid angle. The matching algorithm relies on the pad indices and can be implemented on an FPGA (with a lookup table) 

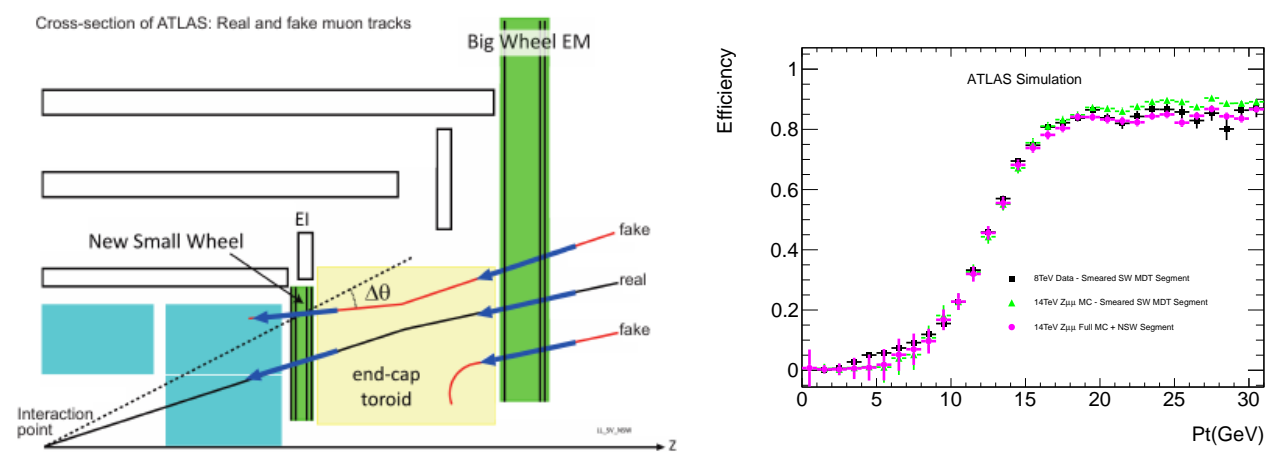

Figure 1: Left: illustration of the NSW location within the ATLAS detector, and of the L1 triggers due to tracks not originating from the interaction point. Right: muon trigger efficiency in the NSW region with the inclusion of the sTGC detector.

located on the detector. Within each sector $\left(1 / 16^{\text {th }}\right.$ of the wheel), two pad triggers are required, one for each multiplet. When they are in matching locations, a 20-strip band is read out by the trigger data serializer (TDS). These data are sent from the detector to the cavern, where the charge centroid is computed. From charge centroids with $\sim 100 \mu \mathrm{m}$ spatial precision, a linear fit is performed, and a segment is reconstructed with $1 \mathrm{mrad}$ angular resolution. The segment is required to point in the direction of the detector center. The sector-trigger candidate is then sent to the sector logic, where the matching between small wheel and big wheel is performed.

\section{Outlook}

The from expected performance of the sTGC-based L1 ATLAS muon trigger upgrade is determined from testbeam studies, from extrapolation of data recorded with the existing detector at $8 \mathrm{TeV}$, and from simulation. As illustrated in figure 1, the NSW can actually provide a L1 muon trigger that is effective at maintaining a low- $p_{T}$ threshold in the forward region when the LHC will operate at its design luminosity $\mathrm{L}=2 \times 10^{34} \mathrm{~cm}^{-2} \mathrm{~s}^{-1}$ [1]. The required performance can be achieved with an 8-layer NSW producing the trigger decision from the pad and strip readouts. The NSW trigger decision will allow a track match with the big wheel at L1, significantly reducing the fake rate. Very good efficiency (93\%) can be achieved, with small dependence on rapidity and on $p_{T}$. The sTGC measurement, combined with the one from the micromegas, will also improve the offline muon spectromenter precision. The ATLAS collaboration has recently approved the NSW project [2], and its construction will begin shortly, on time for its installation in 2018.

\section{References}

[1] The ATLAS collaboration, Letter of Intent for the Phase-I Upgrade of the ATLAS Experiment, CERN-LHCC-2011-012, 2011

[2] The ATLAS collaboration, The New Small Wheel Technical Design Report, CERN-LHCC-2013-006, 2013 